\title{
Dual-energy CT based monitoring of treatment-induced bone marrow changes in lung cancer patients: preliminary results
}

\author{
Sebastian Werner ${ }^{1}$, Bernhard Krauss ${ }^{2}$, Marius Horger ${ }^{3}$ \\ ${ }^{1}$ Department of Diagnostic and Interventional Radiology, Eberhard-Karls-University, Tuebingen, Germany; ${ }^{2}$ Siemens Healthcare GmbH, Diagnostic \\ Imaging, Forchheim, Germany; ${ }^{3}$ Department of Diagnostic and Interventional Radiology, Eberhard-Karls-University, Tuebingen, Germany
}

Contributions: (I) Conception and design: M Horger; (II) Administrative support: None; (III) Provision of study materials or patients: M Horger, B Krauss; (IV) Collection and assembly of data: S Werner, B Krauss; (V) Data analysis and interpretation: S Werner; (VI) Manuscript writing: All authors; (VII) Final approval of manuscript: All authors.

Correspondence to: Sebastian Werner, MD. Department of Diagnostic and Interventional Radiology, Eberhard-Karls-University, Hoppe-Seyler-Str.3, 72076 Tuebingen, Germany. Email: sebastian.werner@med.uni-tuebingen.de.

Background: Systemic lung cancer treatment-induced changes in bone marrow attenuation assessed via dual-energy CT-based virtual non-calcium (VNCa) imaging of the axial skeleton and their relationship to hematological laboratory have not yet been investigated.

Methods: VNCa bone marrow images of the axial skeleton derived from 93 unenhanced reduced dose dual-energy CTs of the thorax and abdomen of 31 patients were retrospectively analyzed. Each patient had received one pre-therapy baseline exam and two consecutive follow-up exams (FU1 and FU2) at a mean of 7.7 and 11.7 weeks after start of therapy. Concurrent hematologic laboratory data were available for every exam. Seven regions of interest were placed in the spine and pelvis and mean VNCa bone marrow attenuation was measured. Twenty-two Patients receiving highly myelotoxic treatment (Group A) were compared to 9 patients receiving less toxic substances (Group B).

Results: Median bone marrow attenuation in Group A/Group B was $-31.8 \mathrm{HU}$ (IQR 12.7)/40.6 HU (IQR, 12.2) at baseline, -46.5 HU (IQR, 12.5)/-43.8 HU (15.7) at FU1 and -46.9 HU (IQR, 22.0)/-38.5 HU (IQR, 18.5) at FU2. In both subgroups the reduction of the mean attenuation between baseline and FU1 was statistically significant although in Group A it was more pronounced; no significant difference was found between FU1 and FU2. The differences between Groups were not statistically significant. Leukopenia rates at FU1 and FU 2 were 50\% and 54.5\% in Group A and 0\% and 22\% in Group B. Anemia rates rose from $31.8 \%$ at baseline to $90 \%$ at FU1 and $86.4 \%$ at FU2 in Group A and fell from $77.8 \%$ at baseline to $66.7 \%$ at FU1 and further to $55.6 \%$ at FU2 in Group B.

Conclusions: Both highly myelotoxic as well as-to a smaller degree-less myelotoxic systemic therapy led to a significant drop in bone marrow attenuation with no significant tendency towards subsequent elevation irrespective of the treatment's degree of toxicity or the presence of myelosuppression and not even under hematological supportive therapy. The results suggest that in this clinical setting an increase in bone marrow attenuation should be regarded as suspicious for tumor infiltration.

Keywords: Bone marrow; carcinoma, bronchogenic; tomography, x-ray computed; chemotherapy induced myelosuppression

Submitted Jul 11, 2021. Accepted for publication Nov 01, 2021.

doi: 10.21037/qims-21-545

View this article at: https://dx.doi.org/10.21037/qims-21-545 


\section{Introduction}

Lung cancer is one of the leading causes of cancer-related deaths in the western world accounting for an estimated $12.4 \%$ of all new cancer cases diagnosed in the US in 2021 (1). Twenty to thirty percent of non-small cell lung carcinoma (NSCLC) patients present with bone metastases at diagnosis and an additional $35-40 \%$ of cases develop bone metastases during the course of the disease, negatively affecting the overall survival $(2,3)$. Bone metastases in lung cancer are quite variable and can, apart from focal osteoblastic or osteolytic lesions, present as diffuse involvement of the bone marrow (BM), i.e., medullary carcinomatosis.

Diagnosis of diffuse bone marrow involvement is challenging. Standard CT is of limited use in particular with respect to involvement of the axial skeleton where the trabecular architecture of the cancellous bone impedes evaluation of the underlying bone marrow (4). More sophisticated imaging techniques which are better suited include MRI using different sequences including diffusionweighted imaging as well as functional imaging modalities like bone scintigraphy or FDG-PET/CT (5-7). However, bone marrow involvement can be missed even by the latter two imaging techniques due to its diffuse distribution and, in case of bone scintigraphy, the possibility that the bone metabolism is not primarily affected (6). In such cases MRI has advanced to potentially the most useful modality for assessing bone marrow tumor invasion (8). In clinical routine diffuse bone marrow involvement is generally worked up based on evaluation of hematological laboratory parameters (e.g., hematopoietic cell count) which are affected as the bone marrow becomes replaced by tumor cells (9). This approach is unfortunately subject to many errors due to the potential effects of coexisting disorders, paraneoplastic phenomena and systemic treatment including bone marrow stimulating supportive drugs on bone marrow composition (10).

Worldwide CT remains the standard imaging modality in the initial diagnosis and follow-up of lung cancer patients due to its availability, excellent spatial resolution and the fact that many of the newly diagnosed lung cancer cases present already in advanced stages. Multiple publications have shown that dual-energy CT (DECT)-based bone marrow imaging is able to significantly enhance the utility of CT depicting bone marrow changes in a variety of clinical contexts, e.g., trauma-related bone marrow edema as well as plasma cell infiltration in multiple myeloma (11-16). DECT is able to differentiate materials based on their particular $\mathrm{x}$-ray absorption characteristics by highlighting the varying behaviors of materials with different atomic numbers, leading to differential energy-dependent $\mathrm{x}$-ray absorption behaviors (11). This allows for removal of trabecula of the cancellous bone to generate so-called virtual-noncalcium ( $\mathrm{VNCa}$ ) images. In the context of systemically treated lung cancer patients this approach has the potential to improve diagnostic performance, e.g., by detecting early non-osteolytic bone marrow infiltration. Due to the complicated dynamics of bone marrow with multiple factors possibly affecting its composition it is necessary to be familiar with the characteristics of bone marrow in lung cancer patients before and during the course of therapy. These characteristics of non-infiltrated bone marrow and its behaviour under treatment in lung cancer patients, as assessed via DECT based bone marrow (BM) imaging, have not yet been specifically investigated in the literature.

Hence, our study aims at quantifying the temporal course of bone marrow attenuation during different treatment regimens by comparing results with those of hematological laboratory data at conventional follow-up intervals with the focus on exclusion of changes overlapping with those expected with medullary carcinomatosis. We present the following article in accordance with the MDAR and STARD reporting checklists (available at https://dx.doi. org/10.21037/qims-21-545).

\section{Methods}

\section{Patients}

The study was conducted in accordance with the Declaration of Helsinki (as revised in 2013). The study was approved by institutional ethics committee of the University Hospital Tübingen (Registration number 203/2021BO2) and individual consent for this retrospective analysis was waived. We performed a retrospective radiological database search for dual-energy non-contrast-enhanced CTs of the thorax and abdomen of patients diagnosed with lung cancer performed in the time period between July 2015 and September 2019. Criteria for inclusion of patients were as follows: availability of 3 consecutive DECTs (one baseline CT before beginning of first line treatment and two follow-up CTs either during first line treatment or after termination of firstline treatment but before beginning of any secondline systemic treatment); histologically proven lung cancer; systemic lung cancer therapy; availability of 
hematological laboratory data including white blood cells (WBC), neutrophils, hemoglobin, red blood cells (RBC) and platelets at each of the three timepoints. Exclusion criteria were the following: known concurrent second malignant disease; previously received systemic anti-cancer treatment; any concurrent disease known to affect the bone or bone marrow; other conditions potentially affecting bone marrow composition (e.g., severe chronic anemia, chronic respiratory diseases, climbing, diving, obesity); known skeletal metastases.

\section{CT examinational protocol and post-processing}

All CT-examinations were performed on a Dual-Source Dual-Energy 128-slice scanner (SOMATOM Definition Flash, Siemens Healthineers, Forchheim, Germany) with the patients positioned supine, and the arms elevated over the head. The following parameters were used: Tube voltage $100 \mathrm{kVp}$ and $140 \mathrm{kVp}$, collimation width $0.6 \mathrm{~mm}$, table speed $69.6 \mathrm{~mm} / \mathrm{s}$, table feed per rotation $23 \mathrm{~mm}$, pitch factor 0.6 , matrix $512 \times 512$, convolution kernel Q40f, tube current time product $100 \mathrm{mAs}(100 \mathrm{kVp})$ and $77 \mathrm{mAs}(140 \mathrm{kVp})$, tin filter. Additional $1 \mathrm{~mm}$ slices were available for postprocessing.

The dual energy data was post-processed with approved software called "dual-energy bone marrow" on syngo.via VB 30A (Siemens Healthineers). The software is based on a three-material decomposition algorithm which assumes that voxels within the BM contain three different materials with particular x-ray absorption characteristics (fat, soft tissue and calcium/bone mineral), which contribute to the total attenuation within the voxel. The voxel's total attenuation can be separated into a fat and soft-tissue partition and a calcium/bone mineral partition. A description of this process has previously been published by Thomas et al. (15). The software uses the low and high-energy source data as input. Two stacks of DICOM images are created as output: an arithmetic average image of both input series which resembles regular CT images with attenuation (HU) comparable to an image with $120 \mathrm{kV}$, and a series of virtual non-calcium BM images. The following thresholds were set for the three-material decomposition: soft tissue, $57 / 55 \mathrm{HU}$ (low/high kV); fat, -103/87 HU; calcium slope, 1.44.

\section{Image analysis}

Two radiologists ( $\mathrm{MH}, \mathrm{SW})$, one with 27 years and one with 4 years of experience in oncology imaging carried out the image analysis. Commercially available software (Syngo.via VB 30A, Siemens Healthineers) was used. Imaging studies were read together side by side. Evaluation differences between the two readers (e.g., malpositioning of regions of interest (ROI) were resolved by consensus reading. First the readers evaluated the dual-energy mixed CT image (further referred to as standard CT image) data sets to exclude the presence of metastatic bone disease. The readers were blinded towards the clinical scenario (e.g., diagnosis, exam timepoint, treatment).

Subsequently, the same software was used to postprocess the DECT image data as described above under CT examinational protocol and post-processing. The softwaregenerates $\mathrm{VNCa}$ images which are displayed as color-coded maps of the BM. It was possible to adjust the degree of overlay between the color map and the CT image. A circular ROI tool was used to measure the mean absolute $\mathrm{VNCa}$ attenuation. Using sagittal multiplanar reconstructions the ROI circle was placed centrally in the cancellous part of the vertebral body. The ROI area was chosen as large as possible while leaving a sufficiently wide margin towards the surrounding cortex. The ROI tool delivered the mean attenuation in Hounsfield units (HU). In each exam seven ROIs were placed, one at each of the following seven subsites of the axial skeleton: vertebral bodies of the upper thoracic spine (TH 1-4), middle thoracic spine (TH 5-8), lower thoracic spine (TH 9-12), upper lumbar spine (L 1-3) and lower lumbar spine (L 4-5); right dorsal iliac crest and left dorsal iliac crest. Regarding vertebral measurements we refrained from strictly measuring specific vertebrae and defined the above mentioned subsites in order to allow the reader to choose vertebrae that were viable for analysis and avoid those that were affected by any conditions that could possibly interfere with HU measurements (fractures, artefacts, orthopaedic hardware, post-radiation fatty replacement of bone marrow etc.).

\section{Laboratory}

Regarding the hematological laboratory we used the normal reference values provided by our institution's central laboratory: WBC, $(4.1-11.8) \times 10^{3} / \mu \mathrm{L}$ (female), (3.8$10.3) \times 10^{3} / \mu \mathrm{L}$ (male); neutrophiles, $(2.1-7.7) \times 10^{3} / \mu \mathrm{L}$ (female), (1.8-7.0) $\times 10^{3} / \mu \mathrm{L}$ (male); RBC, 4.2-6.2 Mio/ $\mu \mathrm{L}$ (female and male); hemoglobin, 12.0-16.0 g/dL (female), 14.0-18.0 g/ $\mathrm{dL}$ (male); platelets, $(150-450) \times 10^{3} / \mu \mathrm{L}$ (female and male). Severity of anemia was graded according to the World Health Organization's (WHO) recommendations (17). 


\section{Statistical analysis}

Patients were divided into two subgroups based on the degree of toxicity of the systemic therapy. Group A contains patients that received systemic treatment of higher toxicity, i.e., all therapy regimens that include any platinum-based agent. Group B contains patients that received treatments of lower toxicity, i.e., antibody-drug conjugates or tyrosine kinase inhibitors. Furthermore, we assessed three different time points: the baseline exam before the begin of systemic therapy, the first follow-up exam (FU1) and the second follow-up exam (FU2). For statistical analysis we calculated the average $\mathrm{VNCa}$ bone marrow attenuation for each DECT dataset, i.e., the average of the $\mathrm{VNCa}$ attenuation values measured via the seven ROIs.

Statistical analysis was carried out using dedicated computer software (IBM Corp. Released 2019. IBM SPSS Statistics for Windows, Version 26.0. Armonk, NY: IBM Corp). $\mathrm{P}<0.05$ was indicative of statistical significance. The Shapiro-Wilk test was used to check for normal distribution of data. In case of multiple comparisons between subgroups $\mathrm{P}$ values were adjusted using Bonferroni correction. In descriptive statistics the mean and standard deviation (SD) are provided for normally distributed data and the median and interquartile range (IQR) for non-normally distributed data.

We tested for interactions between the factors timepoint and therapy subgroup regarding $\mathrm{VNCa}$ attenuation and multiple hematological laboratory parameters (WBC, neutrophiles, RBC, hemoglobin, platelets). This was done via mixed ANOVA. The Huynh-Feldt adjustment was used to correct for violations of sphericity. To assess homogeneity of the error variances, we used Levene's test. To assess homogeneity of covariances Box's test was performed. In case of no statistically significant interaction additional one-way ANOVA to test for main effects of the intersubject factor (differences between therapy groups) and repeated measures ANOVA for main effects of the intersubject factor (differences between timepoints) were performed. Correlations between VNCa attenuation and hematological parameters were assessed via Pearson's correlation coefficient.

\section{Results}

In the time period between July 2015 and September 2019 we identified 315 lung cancer patients with one or more CT examinations, each including the thorax and abdomen.
After the application of the inclusion and exclusion criteria 284 of those patients were excluded, 117 of which due to the presence of bone metastases in their baseline or any of their follow-up CTs. We were able to identify 31 eligible patients. Mean patient age at baseline was 65.9 years with a range of $46.9-83.7$ years. Twelve patients (38.7\%) were female.

Twenty-two patients (71\%) fell into Group A. The median age in Group A was 64.5 years (range, 46.9-83.7 years), eight patients (36\%) were female. Nine patients (29\%) fell into Group B with a median age of 69.4 years (range, 51.982.2 years). Four of the patients (44\%) in Group B were female. There was no statistically significant age difference between the two groups (independent samples $t$-test; $\mathrm{P}=0.167)$. In Group A the median duration of therapy was 15.1 weeks (IQR, 3.4), in Group B it was 29.7 weeks (IQR, 16.9). The median time interval between the start of therapy and the first follow-up exam was 7.7 weeks (IQR, 4.4) in Group A and 11.7 weeks (IQR, 1.7) in Group B. The median time interval between start of therapy and the second follow-up exam was 17.2 weeks (IQR, 7.0) in Group A and 27.9 weeks (IQR, 6.2) in Group B. In Group A 8 of 22 patients (36.4\%) received hematological supportive therapy in the form of leukocyte reduced red blood cells (7 patients) and/or recombinant human erythropoietin (3 patients) and/or granulocyte colony-stimulating factor (1 patient). No patient in Group B received any hematological supportive therapy. Two patients in Group A had received radiotherapy at the time of their baseline exam. An overview regarding treatment for each patient is given in Table 1. Example images of two patients are provided in Figures 1,2.

\section{VNCa attenuation-differences related to therapy subgroup and timepoint}

The median VNCa bone marrow attenuation at baseline in Group A was $-31.8 \mathrm{HU}$ (IQR, 12.7) compared to $-40.6 \mathrm{HU}$ (IQR, 12.2) in Group B. The respective values at FU1 were $-46.5 \mathrm{HU}$ (IQR, 12.5) and $-43.8 \mathrm{HU}(15.7)$. At FU2 we found $-46.9 \mathrm{HU}$ (IQR, 22.0) and $-38.5 \mathrm{HU}$ (IQR, 18.5). Regarding VNCa attenuation mixed ANOVA did not reveal a statistically significant interaction between the examination timepoint and the therapy subgroup $[$ Huynh-Feldt $\mathrm{F}(1.652,47.913)=0.544, \mathrm{P}<0.551$, partial $\left.\eta^{2}=0.018\right]$. Additional one-Way ANOVA did not reveal a statistically significant difference between the therapy subgroups $(\mathrm{P}<0.895)$. Repeated measures ANOVA showed 
Table 1 Therapy overview

\begin{tabular}{|c|c|c|c|c|c|c|}
\hline $\begin{array}{l}\text { Therapy } \\
\text { group }\end{array}$ & $\begin{array}{l}\text { Consecutive } \\
\text { patient number }\end{array}$ & Diagnosis & Therapy & $\begin{array}{c}\text { Duration of } \\
\text { therapy (weeks) }\end{array}$ & Supportive therapy & Radiotherapy \\
\hline \multirow[t]{13}{*}{ Group A } & 21 & SCLC & Carboplatin, etoposid & 16.3 & - & \\
\hline & 2 & NSCLC & Carboplatin, gemcitabin & 15.1 & IrRBC & + \\
\hline & 12 & NSCLC & Carboplatin, paclitaxel & 20.1 & IrRBC, EPO & \\
\hline & 16 & NSCLC & Carboplatin, paclitaxel & 12.0 & - & \\
\hline & 18 & NSCLC & Carboplatin, paclitaxel & 15.0 & - & \\
\hline & 19 & NSCLC & Carboplatin, paclitaxel & 13.0 & - & \\
\hline & 4 & NSCLC & Carboplatin, paclitaxel, atezolizumab & 19.0 & - & \\
\hline & 9 & NSCLC & Carboplatin, paclitaxel, atezolizumab & 16.0 & - & \\
\hline & 14 & NSCLC & Carboplatin, paclitaxel, atezolizumab & 17.4 & - & \\
\hline & 22 & NSCLC & Carboplatin, pemetrexed & 15.0 & IrRBC & \\
\hline & 6 & NSCLC & Carboplatin, pemetrexed & 10.1 & IrRBC, GCSF & \\
\hline & 7 & NSCLC & Carboplatin, pemetrexed & 15.0 & - & + \\
\hline & 10 & NSCLC & Carboplatin, pemetrexed & 15.1 & - & \\
\hline \multirow{8}{*}{ Group B } & 29 & NSCLC & Crizotinib & 29.7 & - & \\
\hline & 27 & NSCLC & Dabrafenib, trametinib & 33.3 & - & \\
\hline & 28 & NSCLC & Osimertinib & 28.1 & - & \\
\hline & 30 & NSCLC & Osimertinib & 27.7 & - & \\
\hline & 20 & NSCLC & Pembrolizumab & 91.6 & - & \\
\hline & 23 & NSCLC & Pembrolizumab & 44.6 & - & \\
\hline & 24 & NSCLC & Pembrolizumab & 23.0 & - & \\
\hline & 31 & NSCLC & Pembrolizumab & 10.3 & - & \\
\hline
\end{tabular}

IrRBC, leucocyte reduced red blood cells; EPO, erythropoietin; GCSF, granulocyte colony-stimulating factor. 


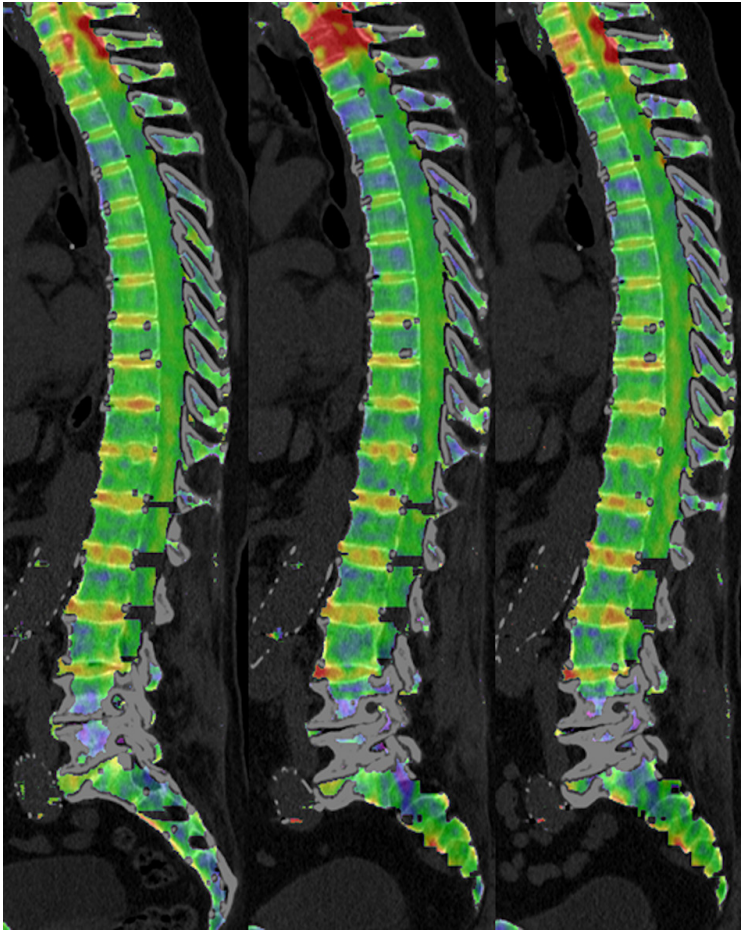

Figure 1 A 71-year-old male patient with non-small cell lung cancer. The patient received 6 cycles of carboplatin, nab-paclitaxel and atezolizumab over 6 months. The figure shows sagittal multiplanar reconstructions of CT images with overlain colorcoded virtual non-calcium maps at baseline (left), first followup (middle) 9 weeks and second follow-up (right) 15 weeks after start of therapy. Bone marrow is represented in green and blue with green indicating higher and blue indicating lower HU values. First follow-up shows increasing patchy blue bone marrow areas signifying fatty conversion which slightly decrease at second follow-up.

a statistically significant effect of the time point on VNCa both in Group B [Huynh-Feldt F(2.000, 16.000) $=5.252, \mathrm{P}=0.018$, partial $\left.\eta^{2}=0.396\right]$, as well as in Group A [Huynh-Feldt $\mathrm{F}(1.449,30.427)=18.553, \mathrm{P}<0.001$, partial $\left.\eta^{2}=0.469\right]$. In Group B there was a significant difference in VNCa attenuation between baseline and FU1 (10.535, $\mathrm{P}=0.040)$. In Group A there was a significant difference between baseline and FU1 $(14.539, \mathrm{P}<0.001)$ as well as between baseline and FU2 (13.014, P=0.003). In both Group A and Group B there was no significant difference between FU1 and FU2.

The median VNCa attenuation of the 8 patients in Group A that had received supportive therapy were -40.8 (IQR 13.0) at baseline, -51.0 (IQR 12.9) at FU1 and -45.7

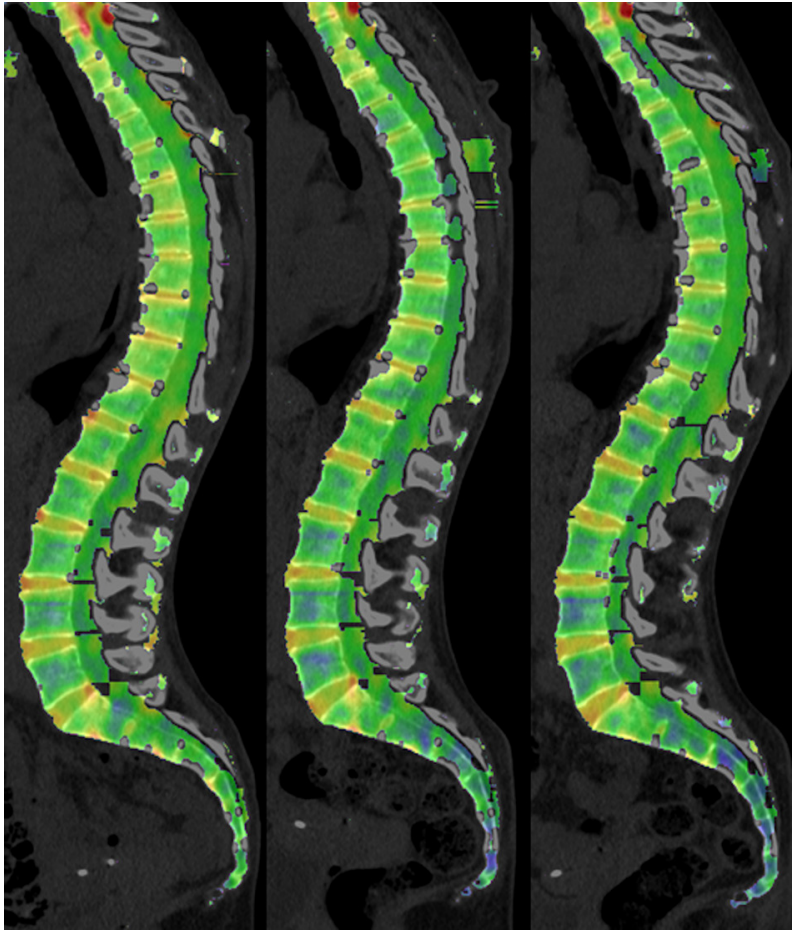

Figure 2 A 59-year-old female patient with non-small cell lung cancer. The patient received Crizotinib until second follow-up and beyond. The figure shows sagittal multiplanar reconstructions of CT images with overlain color-coded virtual non-calcium maps at baseline (left), first follow-up (middle) 11 weeks and second follow-up (right) 29 weeks after start of therapy. Bone marrow is represented in green and blue with green indicating higher and blue indicating lower HU values. First follow-up shows slightly increasing patchy blue bone marrow areas signifying fatty conversion most pronounced in the lumbar spine which does not change significantly at second follow-up.

(IQR 29.1) at FU2.

\section{Hematologic laboratory—differences related to therapy subgroup and timepoint and correlations with VNCa attenuation}

At baseline none of the patients suffered from leukopenia. At FU1 11 out of 22 patients (50\%) in Group A showed leukopenia while it was not observed in Group B. At FU2 Group A kept a similar rate of leukopenia with $54.5 \%$, while at this timepoint it was also observed in Group B with $22.2 \%$. Of 18 patients that showed leukopenia at FU1 and/or at FU2 6 patients received supportive therapy in the form of leucocyte reduced red blood cells and/ 
Table 2 Rates of leukopenia, anemia and thrombopenia at baseline, first (FU1) and second follow-up (FU2)

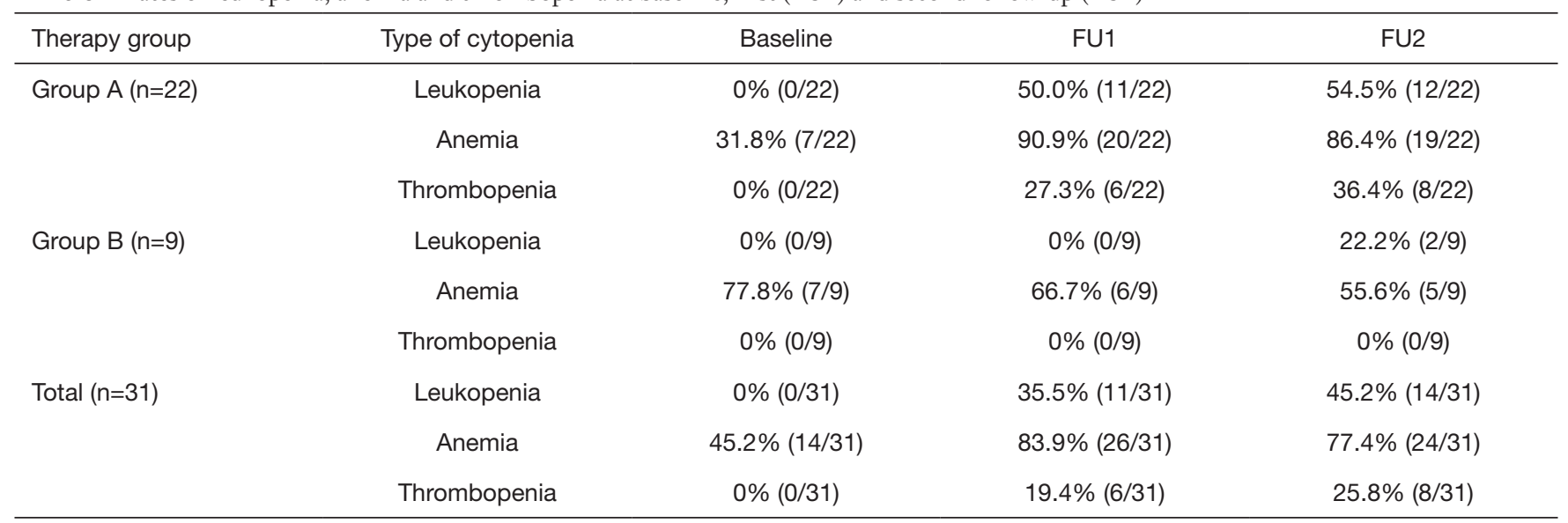

Anemia is defined as $\mathrm{Hb}<12 \mathrm{~g} / \mathrm{dL}$ for women and $\mathrm{Hb}<13 \mathrm{~g} / \mathrm{dL}$ for men according to WHO definition. Leukopenia and Thrombopenia signify values below the lower limit reference value as presented under Laboratory in the Materials and Methods section.

or EPO (erythropoietin); none of these patients received granulocyte colony-stimulating factor (GCSF). The only patient who received GCSF did not show leukopenia at FU1 or FU2, presumably as a consequence of the supportive therapy.

Anemia was observed in both therapy groups already at baseline with 7 out of 22 patients (31.8\%) in Group A and 7 out of 9 patients (77.8\%) in Group B, which was mild in 13 cases and moderate in 1 case. In Group A anemia rates were higher in comparison to baseline and in comparison to Group B with $90.0 \%$ at FU1 and $86.4 \%$ at FU2. In Group $\mathrm{B}$ anemia rates fell to $66.7 \%$ at $\mathrm{FU} 1$ and further to $55.6 \%$ at FU2. At neither time point did the patients in Group B show low platelet counts, while patients in Group A suffered from thrombopenia in $19.4 \%$ at FU1 and $25.8 \%$ at FU2 (Table 2).

Mixed ANOVA found significant interactions between the exam time point and the therapy subgroups regarding WBC, neutrophiles, RBC and hemoglobin. No significant interaction was found regarding platelets.

There was a significant correlation between $\mathrm{VNCa}$ and WBC at FU1 (Pearson correlation 0.373, P=0.039) and between VNCa and platelet count at FU1 (Pearson correlation $0.495, \mathrm{P}=0.005$ ), although after Bonferroni correction this would only leave the latter as statistically significant. There were no statistically significant correlations between $\mathrm{VNCa}$ attenuation and the other laboratory parameters.

\section{Discussion}

The results of our study show that VNCa bone marrow attenuation in the axial skeleton significantly decreases during the course of first line systemic lung cancer treatment and that this change in attenuation seems to be more pronounced at a higher toxicity level. To our knowledge this is the first study examining systemic cancer treatment related changes in bone marrow density via DECT, providing valuable information that can help in clinical decision-making.

The effects of chemotherapy on the MRI appearance of bone marrow in the first weeks are known. It causes an SI decrease in T1w and an increase in T2w MRI images due to marrow edema in the first few days. Within a week it leads to myelosuppression, i.e., a decrease in marrow cellularity and an increase in yellow marrow. Approximately three to four weeks after therapy hematopoietic marrow recovery occurs in a way opposite to marrow conversion $(18,19)$. Hence, the findings of our study are for the most part not surprising. The bone marrow attenuation at baseline was expectedly relatively low with median values between -31.8 and $-40.6 \mathrm{HU}$ reflecting the mean patient age of 65.9 years in our cohort. Negative attenuation-values are compatible with yellow marrow of the adult enabling differentiation from cell dense bone marrow in case of hematopoietic bone marrow reconversion or tumor infiltration. In Group A bone marrow attenuation dropped significantly below baseline levels at the first follow-up exam a median 8 weeks 
after beginning of firstline therapy. A similar but less pronounced result was observed in Group B after a median 12 weeks. Both groups did not show any significant change in attenuation between the first follow-up and the second which took place at a median 17 and 28 weeks after the start of treatment. Altogether, regarding effects on bone marrow attenuation we did not find a statistically significant interaction between the follow-up time point and the treatment's degree of toxicity. Surprisingly, even in patients temporarily receiving myelosupportive therapies we did not observe a significant change in marrow attenuation. The degree of hematologic toxicity of drugs used in treatment of lung cancer is variable, but most agents applied in our cohort are known for these side effects. Myelosuppression occurs in $25-30 \%$ of patients receiving platinum-derivates, in particular if applied at higher doses, affecting all hematopoietic cell lineages $(20,21)$. Similarly, etoposideinduced myelosuppression is dose related occurring from day 15 to day 22 followed by rapid bone marrow recovery by day 21 (22). Combinations of multiple drugs (e.g., cisplatin or carboplatin + pemetrexed) induce even higher rates of marrow toxicity (23). Similar effects have also been reported for paclitaxel (24). Less frequently, myelosuppression may even occur during treatment with checkpoint inhibitors (e.g., pembrolizumab, atezolizumab) $(25,26)$. The trend towards continuous fatty transformation of the bone marrow in Group A could suggest long-lasting or even irreversible myelosuppression by these drugs, although this is difficult to confirm in this study as most patients received additional second line treatment immediately after the investigated time period. The observed changes in bone marrow attenuation are less pronounced than but nevertheless reflected in and supported by the rates of leukopenia, anemia and thrombopenia. Cell counts were clearly more effected by the more toxic treatment in Group A with approximately half of the patients developing persistent leukopenia and high rates of persistent anemia. In Group B leukopenia developed not until the second followup and only in approximately a fifth of the patients, while anemia rates even decreased during the course of therapy. Thrombopenia developed only in Group A and rates were relatively low.

To our knowledge chemotherapy-induced effects on the CT-attenuation of normal bone marrow have not yet been investigated. A direct comparison to the existing literature is difficult due to differences in patient characteristics, therapy regimes and measurement techniques. Carmona et al. (27) performed MRI-based proton density fat fraction measurements of the spine during (i.e., mean $24 \pm 10$ days after baseline) and post (i.e., $55 \pm 13$ days after baseline) chemoradiation therapy of the pelvic region in 19 patients. They did find a significant increase in fat fraction in the directly radiated pelvic and lower lumbar region as well as in the upper lumbar and lower thoracic region that had received a lesser dose. Interestingly they did not find an increase in the upper spinal region that had not received any radiation dose, not even in patients receiving highly myelotoxic chemotherapy. Altehoefer et al. (28) evaluated bone marrow changes via MRI in 14 patients undergoing high-dose chemotherapy and peripheral blood stem cell transplantation for breast cancer. They found a significant signal intensity decrease on $\mathrm{T} 1 \mathrm{w}$ images and an increase on STIR images during induction chemotherapy which was interpreted as acute marrow edema. Six to eight weeks after high dose chemotherapy and reinfusion of stem cells T1w signal intensity returned to baseline levels while STIR signal intensity dropped to levels below the baseline. They interpret these findings as signifying therapy-associated marrow edema followed by deposition of paramagnetic substances or small amounts of fibrosis in terms of benign posttherapeutic changes. Bolan et al. (29) measured bone marrow fat fraction via MRI in the femoral necks and L4 vertebrae in 13 women with gynecologic malignancies receiving radiation and/or chemotherapy. Six months after therapy fat fraction had increased a median $16.2 \%$ in the L4 vertebrae and $4.5 \%$ in the femoral necks. After 12 months fat fraction seemed to further increase although they were not able to statistically verify this. MRIbased quantification of active bone marrow in the pelvic region can also act as a useful tool in predicting levels of myelosuppression which recently has been shown by Kuncman et al. (30) in a cohort of rectal cancer patients receiving chemoradiotherapy.

Due to the retrospective nature of the study our patient cohort is relatively small and shows some heterogeneity regarding the time of follow-up and the treatment regimens. These limitations give this investigation the character of a feasibility study with preliminary results. The goal of a follow-up study should be to include a higher number of patients with a larger degree of homogeneity regarding the timing of follow-up CT exams. Furthermore, we must acknowledge that the presence of bone metastases was excluded on CT images. It is however possible that some patients would have shown signs of bone metastases on more sensitive imaging modalities like MRI or FDG-PET/CT. Nevertheless, the fact that 
none of the patients showed any demarcation of bone lesions under systemic therapy provides some reassurance that there had not been any metastases at baseline. Two patients in Group A had received radiation therapy and we cannot fully exclude that this had an effect even on bone marrow outside the radiation field. Lastly, images were read in consensus and thus an analysis of inter- and intrareader reliability, which is important for a quantitative analysis with multiple follow-ups, was not performed and should be part of a follow-up study. In conclusion, both highly myelotoxic as well as-to a smaller degree-less myelotoxic systemic therapy led to a significant drop in bone marrow attenuation with no significant tendency towards subsequent elevation irrespective of the treatment's degree of toxicity or the presence of myelosuppression and not even following hematological supportive therapy. This suggests that if present an increase in bone marrow attenuation in this clinical setting should be considered suspicious of tumor cell marrow invasion possibly requiring histologic diagnosis.

\section{Acknowledgments}

Funding: None.

\section{Footnote}

Reporting Checklist: The authors have completed the MDAR and STARD reporting checklist. Available at https://dx.doi. org/10.21037/qims-21-545

Conflicts of Interest: All authors have completed the ICMJE uniform disclosure form (available at https://dx.doi. org/10.21037/qims-21-545). BK reports that he is a current employee of Siemens Healthcare GmbH. The other authors have no conflicts of interest to declare.

Ethical Statement: The authors are accountable for all aspects of the work in ensuring that questions related to the accuracy or integrity of any part of the work are appropriately investigated and resolved. The study was conducted in accordance with the Declaration of Helsinki (as revised in 2013). The study was approved by institutional ethics committee of the University Hospital Tübingen (Registration number 203/2021BO2) and individual consent for this retrospective analysis was waived. Where illustrations include recognizable individuals, living or deceased, great care was taken to ensure that consent for publication has been given. Patient anonymity was preserved. Photographs were cropped sufficiently to prevent human subjects from being recognized and the eyes and eyebrows (at a minimum) were masked using Coarse Pixilation to make the individual unrecognizable.

Open Access Statement: This is an Open Access article distributed in accordance with the Creative Commons Attribution-NonCommercial-NoDerivs 4.0 International License (CC BY-NC-ND 4.0), which permits the noncommercial replication and distribution of the article with the strict proviso that no changes or edits are made and the original work is properly cited (including links to both the formal publication through the relevant DOI and the license). See: https://creativecommons.org/licenses/by-nc-nd/4.0/.

\section{References}

1. National Cancer Institute: Surveillance, Epidemiology and End Results (SEER) Program. Cancer Stat Facts: Lung and Bronchus Cancer. 2021. Available online: https:// seer.cancer.gov/statfacts/html/lungb.html. Accessed 24.10.2021.

2. Roodman GD. Mechanisms of bone metastasis. $\mathrm{N}$ Engl J Med 2004;350:1655-64.

3. Santini D, Barni S, Intagliata S, Falcone A, Ferraù F, Galetta D, et al. Natural History of Non-Small-Cell Lung Cancer with Bone Metastases. Sci Rep 2015;5:18670.

4. Horger M, Kanz L, Denecke B, Vonthein R, Pereira P, Claussen CD, Driessen C. The benefit of using wholebody, low-dose, nonenhanced, multidetector computed tomography for follow-up and therapy response monitoring in patients with multiple myeloma. Cancer 2007;109:1617-26.

5. Chen YW, Huang MY, Hsieh JS, Hou MF, Chou SH, Lin CL. Discordant findings of skeletal metastasis between tc 99M MDP bone scans and F18 FDG PET/CT imaging for advanced breast and lung cancers--two case reports and literature review. Kaohsiung J Med Sci 2007;23:639-46.

6. Imamura F, Kuriyama K, Seto T, Hasegawa Y, Nakayama T, Nakamura Si, Horai T. Detection of bone marrow metastases of small cell lung cancer with magnetic resonance imaging: early diagnosis before destruction of osseous structure and implications for staging. Lung Cancer 2000;27:189-97.

7. Raynor WY, Al-Zaghal A, Zadeh MZ, Seraj SM, Alavi A. Metastatic Seeding Attacks Bone Marrow, Not Bone: Rectifying Ongoing Misconceptions. PET Clin 
2019;14:135-44.

8. Schmidt GP, Schoenberg SO, Schmid R, Stahl R, Tiling R, Becker CR, Reiser MF, Baur-Melnyk A. Screening for bone metastases: whole-body MRI using a 32-channel system versus dual-modality PET-CT. Eur Radiol 2007;17:939-49.

9. de Castria TB, da Silva EM, Gois AF, Riera R. Cisplatin versus carboplatin in combination with third-generation drugs for advanced non-small cell lung cancer. Cochrane Database Syst Rev 2013;(8):CD009256.

10. Minutoli F, Pergolizzi S, Blandino A, Mormina E, Amato E, Gaeta M. Effect of granulocyte colony-stimulating factor on bone marrow: evaluation by intravoxel incoherent motion and dynamic contrast-enhanced magnetic resonance imaging. Radiol Med 2020;125:280-7.

11. Gosangi B, Mandell JC, Weaver MJ, Uyeda JW, Smith SE, Sodickson AD, Khurana B. Bone Marrow Edema at Dual-Energy CT: A Game Changer in the Emergency Department. Radiographics 2020;40:859-74.

12. Kosmala A, Weng AM, Krauss B, Knop S, Bley TA, Petritsch B. Dual-energy CT of the bone marrow in multiple myeloma: diagnostic accuracy for quantitative differentiation of infiltration patterns. Eur Radiol 2018;28:5083-90.

13. Kosmala A, Weng AM, Heidemeier A, Krauss B, Knop S, Bley TA, Petritsch B. Multiple Myeloma and DualEnergy CT: Diagnostic Accuracy of Virtual Noncalcium Technique for Detection of Bone Marrow Infiltration of the Spine and Pelvis. Radiology 2018;286:205-13.

14. Goo HW, Goo JM. Dual-Energy CT: New Horizon in Medical Imaging. Korean J Radiol 2017;18:555-69.

15. Thomas C, Schabel C, Krauss B, Weisel K, Bongers M, Claussen CD, Horger M. Dual-energy CT: virtual calcium subtraction for assessment of bone marrow involvement of the spine in multiple myeloma. AJR Am J Roentgenol 2015;204:W324-31.

16. Simons D, Kachelriess M, Schlemmer HP. Recent developments of dual-energy CT in oncology. Eur Radiol 2014;24:930-9.

17. WHO. Haemoglobin concentrations for the diagnosis of anaemia and assessment of severity. Vitamin and Mineral Nutrition Information System. World Health Organization, Geneva. 2011. Available online: http:// www.who.int/vmnis/indicators/haemoglobin.pdf. Accessed 24/10/2021.

18. Daldrup-Link HE, Henning T, Link TM. MR imaging of therapy-induced changes of bone marrow. Eur Radiol 2007;17:743-61.
19. Ollivier L, Gerber S, Vanel D, Brisse H, Leclère J. Improving the interpretation of bone marrow imaging in cancer patients. Cancer Imaging 2006;6:194-8.

20. U.S. Food and Drug Administration. PARAPLATIN Label. U.S. Food and Drug Administration. 2010. Available online: https://www.accessdata.fda.gov/ drugsatfda_docs/label/2010/020452s005lbl.pdf. Accessed 29.12.2020.

21. U.S. Food and Drug Administration. PLATINOL label. 2010. Available online: https://www.accessdata.fda.gov/ drugsatfda_docs/label/2011/018057s080lbl.pdf. Accessed 29.12.2020.

22. U.S. Food and Drug Administration. ETOPOPHOS label. 2010. Available online: https://www.accessdata. fda.gov/drugsatfda_docs/label/2011/020457s013lbl.pdf. Accessed 29.12.2020.

23. U.S. Food and Drug Administration. ALIMTA label. 2018. Available online: https://www.accessdata.fda.gov/ drugsatfda_docs/label/2018/021462s051lbl.pdf. Accessed 29.12.2020.

24. U.S. Food and Drug Administration. TAXOL label. 2011. Available online: https://www.accessdata.fda.gov/ drugsatfda_docs/label/2011/020262s049lbl.pdf. Accessed 29.12.2020.

25. U.S. Food and Drug Administration. TECENTRIQ label. 2019. Available online: https://www.accessdata.fda.gov/ drugsatfda_docs/label/2019/761034s018lbl.pdf. Accessed 29.12.2020.

26. U.S. Food and Drug Administration. KEYTRUDA label. 2019. Available online: https://www.accessdata.fda.gov/ drugsatfda_docs/label/2019/125514s040lbl.pdf. Accessed 29.12.2020.

27. Carmona R, Pritz J, Bydder M, Gulaya S, Zhu H, Williamson CW, Welch CS, Vaida F, Bydder G, Mell LK. Fat composition changes in bone marrow during chemotherapy and radiation therapy. Int J Radiat Oncol Biol Phys 2014;90:155-63.

28. Altehoefer C, Laubenberger J, Lange W, Kraus A, Allmann KH, Uhrmeister P, Langer M. Prospective evaluation of bone marrow signal changes on magnetic resonance tomography during high-dose chemotherapy and peripheral blood stem cell transplantation in patients with breast cancer. Invest Radiol 1997;32:613-20.

29. Bolan PJ, Arentsen L, Sueblinvong T, Zhang Y, Moeller S, Carter JS, Downs LS, Ghebre R, Yee D, Froelich J, Hui S. Water-fat MRI for assessing changes in bone marrow composition due to radiation and chemotherapy in gynecologic cancer patients. J Magn Reson Imaging 
2013;38:1578-84.

30. Kuncman Ł, Stawiski K, Masłowski M, Kucharz J, Fijuth J. Dose-volume parameters of MRI-based active bone marrow predict hematologic toxicity of chemoradiotherapy for rectal cancer. Strahlenther Onkol 2020;196:998-1005.

Cite this article as: Werner S, Krauss B, Horger M. Dualenergy CT based monitoring of treatment-induced bone marrow changes in lung cancer patients: preliminary results. Quant Imaging Med Surg 2022;12(3):1871-1881. doi: 10.21037/ qims-21-545 